\title{
Can Tomosynthesis Solve the Problem of Mammographically Indeterminate Breast Lesions?
}

\author{
OMNIA MOKHTAR, M.D.*; SAHAR MAHMOUD, M.D.** and AMR KAMAL, M.D.*** \\ The Department of Radiology, National Cancer Institute of Cairo*, Faculty of Medicine, Beni-Suef University** and \\ Surgery Department***, National Cancer Institute of Cairo, Egypt
}

\begin{abstract}
Background: Breast cancer in women is a major public health problem throughout the world. To reduce the morbidity and mortality associated with breast cancer, early detection becomes a very important job. Addition of breast Tomosynthesis to mammography examination reduced and eliminated tissue overlap especially in women with radiographically dense breast.
\end{abstract}

Aim of Study: To evaluate the impact of adding Digital Breast Tomosynthesis (DBT) to Full Field Digital Mammography (FFDM) in detection, characterization and diagnosis of mammographically indeterminate breast lesions.

Subjects and Methods: The study included 80 patients with 86 indeterminate findings on mammography. DBT examination was done and imaging findings were evaluated before and after the use of DBT.

Results: Tomosynthesis showed better diagnostic performance compared to mammography. The sensitivity of Tomosynthesis was $90 \%$, the specificity was $91.1 \%$, the positive predictive value of $88 \%$, the negative predictive value was $92.7 \%$ and the diagnostic accuracy was $91 \%$, while the sensitivity of mammography was $62.5 \%$, the specificity was $59 \%$, the positive predictive value was $52.1 \%$, the negative predictive value was $68.8 \%$, and the diagnostic accuracy was $60.4 \%$.

Conclusion: DBT significantly enhanced the detection and characterization of indeterminate breast lesions on digital mammography especially in the context of dense breast parenchyma.

Key Words: Digital mammography - Tomosynthesis - Indeterminate breast lesions.

\section{Introduction}

BREAST cancer in women is considered a major public health problem throughout the world. It is the most common cancer among women both in developed and developing countries, accounting for $22.9 \%$ of all female cancers. In Egypt, breast cancer accounts for $37.7 \%$ of the total new cancer

Correspondence to: Dr. Omnia Mokhtar, The Department of Radiology, National Cancer Institute of Cairo, Egypt cases. It is the leading cause of cancer related mortality accounting for $29.1 \%$ of the cancer related deaths [1].

Early detection of breast cancer is an important task to reduce the morbidity and mortality associated with breast cancer cases [2]. Mammography is the basic breast imaging modality for early detection and diagnosis of breast cancer [3]

Full Field Digital Mammography developments have been rapid, enabling high-quality breast images with higher contrast resolution, an improved dynamic range, and rapid processing of data and images when compared with Screen Film Mammography. However, some limitations still persist [4]

Mammography has low sensitivity and specificity in women with radiographically dense breast due to decrease contrast between a possible tumor and surrounding breast tissue and summation of tissues may obscure lesions [5].

Breast Tomosynthesis by reducing or eliminating tissue overlap can markedly solve most of these problems.

So, the aim of our study was to evaluate the impact of adding digital breast Tomosynthesis (DBT) to Full Field Digital Mammography (FFDM) in detection, characterization and diagnosis of mammographically indeterminate breast lesions.

\section{Patients and Methods}

A prospective study was performed in Female Imaging Unit of the National Cancer Institute (NCI), Cairo University, between April 2018 and November 2018, the study was approved by its Research and Ethical Committee with informed patients consent. 
The study was conducted on 80 patients their ages range from $35-75$ presented with palpable breast mass or detected incidentally in screening program.

\section{Inclusion criteria:}

1 - Female of age group $\geq 30$ years.

2- Indeterminate breast lesions by mammography (BIRADS III, IV).

3- Dense breast in patients with complain (BIRADS 0 ) which need further assessment.

\section{Patient exclusion criteria:}

1- Pregnant female to avoid the hazards of ionizing radiation to the fetus.

2- Tender breast that can't tolerate compression.

3- Patients who mammographic BIRADS other than 0 , III and IV.

All cases with indeterminate digital mammography lesions underwent DBT.

\section{Patients' preparations:}

Full personal, past and family history of the patients were taken including age, previous mammograms, prior surgeries, complains if present, superficial marks (such as prominent moles, scars from an incision), family history of breast cancer and history of hormonal pills.

\section{Imaging technique:}

Mammographic examination was performed using full field digital mammography machine with DBT (Senographe Essential GE healthcare and Hologic Selenia dimension 2D/3D). For digital mammography; two views for each breast (CC) and (MLO) were taken. For Tomosynthesis also $\mathrm{CC}$ and MLO series views were taken.

For digital mammography the entire procedure including the patients' preparation about 10 minutes while each exposure took 20 seconds, Tomosynthesis is slower each arc projection took about 1 minute.

Three Dimensional DBT involved the acquisition of twelve to fifteen $2 \mathrm{D}$ projection exposures by a digital detector from a mammographic X-ray source which moves over a limited arc angle. The $3 \mathrm{D}$ volume of compressed breast was reconstructed from the $2 \mathrm{D}$ projections in the form of series of images (slices) through the entire breast. Images were assessed on the workstation.

The results of both mammography studies; Digital mammography and Tomosynthesis for each patient were compared in terms of detection, visual accuracy, main radiological features, sensitivity, and specificity and BIRADS classification.

Image analysis and interpretation of Mammography and $3 D$ Digital Tomosynthesis:

- Breast density was assessed for each patient.

- Each lesion was evaluated regarding site, type (mass, focal asymmetry \pm calcifications and size).

- Lesions were classified as benign or malignant according to the mammography BI-RADS lexicon morphology descriptors.

- Mass lesions: Shape, margin, density and size.

- Asymmetry: Simple, focal, global or developing.

- Calcifications: Morphology and distribution.

- Both modalities were independently reported as a part of the diagnostic procedure, we determined the BI-RADS category of the lesions in each of the 2 imaging modalities individually according to the BI-RADS lexicon 2013 classification, guided by the results of mammographic findings.

All our cases underwent pathological confirmation (by surgical biopsy, mastectomy, us guided biopsy or stereotactic biopsy) and/or follow-up.

\section{Results}

We performed our study on 80 female patients with 86 lesions; $46(56.3 \%)$ lesions at right and 32 $(40 \%)$ at left breast. Patients were aged from 35 to 75 years old with mean age $46.3 \pm 9.4$ (mean \pm $\mathrm{SD})$.

Half of the patients in our study had first-and second-degree relatives who were positive for breast cancer. Both imaging modalities were interpreted independently, and all breast findings were assessed regarding the presence of mass lesion or any other suspicious abnormalities.

Cases were presenting with; breast lump only 49 cases $(61.3 \%)$, breast lump with breast edema $4(5 \%)$, lump with nipple retraction in $4(5 \%)$ cases and asymptomatic in 23 (28.7\%). Findings detected by mammography and Tomosynthesis were shown in Table (1). Some affected breasts had multiple lesions. DBT detected 10 lesions not seen by DM. characteristics of findings detected by DBT and digital mammography were shown at Tables (25). Distribution and ACR classification of breast density were reported in Table (6). Table (7) revealed that; 10 lesions were clarified by DBT not seen in DM. These lesions were found in dense breast more than non-dense. 
Eighty six lesions were detected by digital mammography, 40 of them were mass and 46 were non mass while 96 lesions were detected by DBT, 76 of them were mass and 20 non mass. Final diagnosis were 82 mass and 14 non mass.

\section{Z-test for proportion:}

Table (8) showed that there were high statistical significant differences between digital mammography and tomosynthesis in mass detection, in all ACR categories. Tomosynthesis had higher sensitivity and accuracy for mass detection than digital mammography, while digital mammography had more false negative rate than tomosynthesis due to overlapping tissue in dense breast which obscuring underlying masses. Comparison between mass detection by digital mammography and tomosynthesis in non-dense breast (ACR A and B categories) (Table 9) showed that there were high statistical significant differences between digital mammography and tomosynthesis in mass detection, in nondense breast. Tomosynthesis had higher sensitivity and accuracy for mass detection than digital mammography, while digital mammography had more false negative rate than tomosynthesis. While in in dense breast (ACR C and D) (Table 10), we found high statistical significant differences between digital mammography and tomosynthesis in mass detection. Tomosynthesis had higher Sensitivity and Accuracy for mass detection than digital mammography, while digital mammography had more false negative rate than tomosynthesis due to overlapping tissue in dense breast which may obscure underlying masses. Final diagnosis was according to histo-pathological analysis of biopsy and surgical samples, fine-needle aspiration cytology or follow-up.

Table (11) showed that $41.7 \%$ of lesions detected by tomosynthesis were malignant tumors, $58.3 \%$ were benign tumors, the most common benign lesions were fibrocystic changes followed by fibroadenomata and most common malignant tumor was invasive ductal carcinoma.

We detected were high statistical significant differences between digital mammography and tomosynthesis in BIRADS as demonstrated in Table (12). Regarding that DBT clarify 10 more lesions not visualized by DM.

There was statistical significant difference in cancer detection between digital mammography and tomosynthesis, in non-dense breast (ACR A and B categories) as well as dense breast (ACR C and $\mathrm{D}$ categories). Tomosynthesis had higher Sensitivity, specificity and accuracy than digital mam- mography, while digital mammography had more false positive rate than tomosynthesis (Tables $13,14)$.

Figs. (1,2) 54-year-old female. (1) Mammographic views, ACR C. Left breast shows areas of focal asymmetry associated with architectural distortion in the upper outer and lower inner aspect of the left breast (BIRADS Iva). No spiculated masses or suspicious micro-calcifications. (2) Tomosynthesis reveals: Left breast two small irregular, ill-defined lesions with spiculated margins seen at upper outer quadrant which measures $10 \times 8$ $\mathrm{mm}$ and lower inner quadrant which measures 9.5 $X 8 \mathrm{~mm}$ associated with axillary LNs one of the with lost fatty hilum (BIRADS V). Histopathology: Invasive ductal carcinoma. Conclusion: 3D digital breast tomosynthesis easily detects two small spiculated lesions at UOQ and LIQ of the left breast. Tomosynthesis upgrades two left breast lesions which proved to be invasive ductal carcinoma.

Figs. $(3,4)$ 56-year-old female. (3) Digital Mammography reveals ACR A. Left breast shows focal asymmetry in the upper outer quadrant (BIRADS III). No spiculated masses or suspicious microcalcifications. (4) Tomosynthesis, left breast shows area of parenchymal distortion associated with small spiculated lesion at upper outer quadrant (BIRADS IVc). Histopathology: Invasive lobular carcinoma. Conclusion: Tomosynthesis easily detects architectural distortion with small spiculated lesion at UOQ of the left breast. Tomosynthesis upgrades the left breast lesion which proved to be invasive lobular carcinoma.

Figs. $(5,6) 52$ year old female. (5) Digital Mammography reveals; ACR B. Right UOQ area of architecture distortion (BIRADS IVa). (6) 3D Tomosynthesis reveals: Right breast show UOQ ill-defined dense mass with irregular outline and spiculated margins measures about $32 \times 24 \mathrm{~mm}$, no suspicious micro-calcifications (BIRADS IVc). Histopathology: Invasive ductal carcinoma. Conclusion: 3D Digital Tomosynthesis upgrades right breast lesion from IVa to IVc which proved to be invasive ductal carcinoma.

Figs. $(7,8) 35$-year-old female complaining from right breast lump. (7) Digital Mammography reveals; ACR D. Right outer central area of architectural distortion (BIRADS IVa). Left enlarged axillary lymph node with preserved fatty hilum. Tomosynthesis reveals: The lesion becomes more clearly define with spiculated margins on 3D Digital Tomosynthesis images measuring about $25 \times 27 \mathrm{~mm}$ 
(BIRADS IVc). Histopathology: Invasive duct carcinoma. Conclusion: The Tomosynthesis has better margin characterization, which easily detects the spiculated margins of this lesion and upgraded the BIRADS category from IVa to IVc.

Figs. $(9,10) 48$ year old female complaining from left breast lump. (9) Digital Mammography reveals; ACR C. Left UOQ dense lesion with obscured margin (BIRADS Iva). No suspicious microcalcific foci. Bilateral axillary LNs with preserved fatty hilum. (10) Tomosynthesis reveals; left UOQ well defined rounded medium dense lesion with smooth margins and minute peripheral calcific foci measures about $16 \mathrm{X} 16 \mathrm{~mm}$ associated with characteristic halo sign BIRADS II. Final diagnosis: Simple cyst. Conclusion: Tomosynthesis better delineate margins of the lesion and presence of calcifications this downgrades BIRADS from being suspicious (IVa) into benign lesion (II) which proved to be simple cyst.

Figs. $(11,12) 36$ year old female complaining from right nipple retraction. (11) Digital Mammography reveals; ACR B. Right nipple retraction with central retro areolar asymmetrical density (BIRADS IVa). Left well defined medium density lesion in UOQ seen in CC view and obscured in MLO due to summation of glandular tissue measuring about $11 \mathrm{X} 12.5 \mathrm{~mm}$. (BIRADS III). 3D Tomosynthesis reveals; right central retro-areolar dense mass with irregular outline and spiculated margins associated with retracted nipple measuring about $35 \times 21 \mathrm{~mm}$ (BIRADS IVc) and left UOQ well defined medium dense lesion with better definition of margins in both CC and MLO views measures about $13 \mathrm{X}$ $11 \mathrm{~mm}$. (BIRADS III). Histopathology: Right invasive lobular carcinoma and left fibroadenoma. Conclusion: Tomosynthesis upgrades right breast lesions from III to IVc with better delineation of its margins which proved to be invasive lobular carcinoma, however, tomosynthesis has the same BIRADS of the left breast lesion as DM with better characterization for its margins which proved to be fibroadenoma.

Table (1): Number of findings in affected breasts detected by mammography, tomosynthesis.

\begin{tabular}{lcc}
\hline Number of findings & $\begin{array}{c}\text { Mammography } \\
\text { No. }(\%)\end{array}$ & $\begin{array}{c}\text { Tomosynthesis } \\
\text { No. }(\%)\end{array}$ \\
\hline Right breast & $49(57)$ & $55(57.3)$ \\
Left breast & $33(38.4)$ & $37(38.5)$ \\
Both breasts & $4(4.6)$ & $4(4.2)$ \\
\hline Total & $86(100)$ & $96(100)$ \\
\hline
\end{tabular}

Table (2): Findings detected by digital mammography.

\begin{tabular}{lcc}
\hline \multirow{2}{*}{$\begin{array}{l}\text { Digital mammography } \\
\text { findings }\end{array}$} & \multicolumn{2}{c}{ Number of findings (n=86) } \\
\cline { 2 - 3 } & No. & $\%$ \\
\hline - Mass & 40 & 46.5 \\
- Asymmetry & 30 & 34.9 \\
- Architecture distortion & 7 & 8.1 \\
- Clusters of micro-calcification & 5 & 5.8 \\
$\quad$ with no underlying mass & & \\
- Dense breast (BIRADS 0) & 4 & 4.7 \\
\hline
\end{tabular}

Table (3): Characteristics of masses detected by digital mammography.

\begin{tabular}{llll}
\hline \multirow{2}{*}{ Mass characteristics } & \multicolumn{2}{c}{ Detected masses $(\mathrm{n}=40)$} \\
\cline { 3 - 4 } & No. & $\%$ \\
\hline Mass margin: Obscured & 19 & 47.5 \\
& Ill-defined & 15 & 37.5 \\
& Well-defined & 6 & 15 \\
Mass shape: & Irregular & 18 & 45 \\
& Round & 12 & 30 \\
& Oval & 5 & 12.5 \\
& Macro lobulated & 5 & 12.5 \\
\hline
\end{tabular}

Table (4): Findings detected by tomosynthesis.

\begin{tabular}{lcc}
\hline \multirow{2}{*}{ Tomosynthesis findings } & \multicolumn{2}{c}{ Number of findings (n=96) } \\
\cline { 2 - 3 } & No. & $\%$ \\
\hline - Mass & 76 & 79.2 \\
- Architecture distortion & 4 & 4.2 \\
- Dilated ducts & 3 & 3.1 \\
- Clusters of micro-calcification & 2 & 2.1 \\
$\quad$ with no underlying mass & & \\
- Asymmetrical densities & 4 & 4.2 \\
- Overlapped glandular tissue & 7 & 7.3 \\
\hline
\end{tabular}

Table (5): Characteristics of masses detected by tomosynthesis.

\begin{tabular}{llcc}
\hline \multirow{2}{*}{ Mass characteristics } & \multicolumn{2}{c}{ Detected masses (n=76) } \\
\cline { 3 - 4 } & No. & $\%$ \\
\hline Mass margins: Well-defined & 34 & 44.7 \\
& Speculated & 30 & 39.5 \\
& Ill-defined & 12 & 15.8 \\
Mass shape: & Irregular & 35 & 46.1 \\
& Round & 25 & 32.9 \\
& Oval & 10 & 13.1 \\
& Macro lobulated & 6 & 7.9 \\
\hline
\end{tabular}

Table (6): Frequency distribution of breast densities of study participants according to ACR BIRADS lexicon.

\begin{tabular}{lll}
\hline \multirow{2}{*}{ ACR Category } & \multicolumn{2}{c}{ Study participant $(\mathrm{n}=80)$} \\
\cline { 2 - 3 } & No. & $\%$ \\
\hline A & 4 & 5 \\
B & 27 & 33.7 \\
C & 42 & 52.5 \\
D & 7 & 8.8 \\
\hline
\end{tabular}


Table (7): Shows number of findings in each breast category detected by digital mammography and digital breas tomosynthesis.

\begin{tabular}{lccc}
\hline $\begin{array}{l}\text { ACR } \\
\text { category }\end{array}$ & $\begin{array}{c}\text { Number of study } \\
\text { participant }\end{array}$ & $\begin{array}{c}\text { No. of findings } \\
\text { by FFDM (86) }\end{array}$ & $\begin{array}{c}\text { No. of findings } \\
\text { by DBT (96) }\end{array}$ \\
\hline A & 4 & 4 & 4 \\
B & 27 & 29 & 30 \\
C & 42 & 46 & 51 \\
D & 7 & 7 & 11 \\
\hline Total & 80 & 86 & 96 \\
\hline
\end{tabular}

Table (8): Comparison between mass detection by digital mammography and tomosynthesis in all ACR categories: Considering that mass as positive and non mas-mass as negative.

\begin{tabular}{llllll}
\hline $\begin{array}{l}\text { Mass } \\
\text { detection }\end{array}$ & $\begin{array}{c}\text { Digital } \\
\text { mammo- } \\
\text { graphy }\end{array}$ & $\begin{array}{c}\text { Tomosyn- } \\
\text { thesis }\end{array}$ & $\mathrm{X}^{2}$ & \multicolumn{1}{c}{$p$} \\
\hline True positive & $40(41.7 \%)$ & $76(79.2 \%)$ & 22.3 & $<0.001$ (HS) \\
False positive & $0(0.0 \%)$ & $0(0.0 \%)$ & $\mathrm{NA}$ & $\mathrm{NA}$ & \\
True negative & $14(14.6 \%)$ & $14(14.6 \%)$ & $\mathrm{NA}$ & $\mathrm{NA}$ \\
False negative & $42(43.7 \%)$ & $6(6.2 \%)$ & 27.6 & $<0.001$ & (HS) \\
Sensitivity & $49 \%$ & $92.7 \%$ & $6.5^{*}$ & $<0.001$ & (HS) \\
Specificity & $100 \%$ & $100 \%$ & $\mathrm{NA}$ & $\mathrm{NA}$ \\
Accuracy & $56.3 \%$ & $93.7 \%$ & $5.9^{*}$ & $<0.001$ (HS) \\
\hline
\end{tabular}

NA: Not Applicable.

Table (9): Comparison between mass detection by digital mammography and tomosynthesis in non-dense breast (ACR A and B categories): Considering that mass as positive and non-mass as negative.

\begin{tabular}{lllll}
\hline $\begin{array}{l}\text { Mass } \\
\text { detection }\end{array}$ & $\begin{array}{c}\text { Digital } \\
\text { mammo- } \\
\text { graphy }\end{array}$ & $\begin{array}{c}\text { Tomosyn- } \\
\text { thesis }\end{array}$ & $\mathrm{x}^{2}$ & $p$ \\
\hline True positive & $21(61.8 \%)$ & $29(85.3 \%)$ & 3.7 & 0.05 \\
False positive & $0(0.0 \%)$ & $0(0.0 \%)$ & $\mathrm{NA}$ & $\mathrm{NA}$ \\
True negative & $4(11.8 \%)$ & $4(11.8 \%)$ & $\mathrm{NA}$ & $\mathrm{NA}$ \\
False negative & $9(26.4 \%)$ & $1(2.9 \%)$ & 27.6 & $0.01(\mathrm{~S})$ \\
Sensitivity & $70.0 \%$ & $96.7 \%$ & $2.8^{*}$ & $<0.004(\mathrm{HS})$ \\
Specificity & $100 \%$ & $100 \%$ & $\mathrm{NA}$ & $\mathrm{NA}$ \\
Accuracy & $73.5 \%$ & $97.1 \%$ & $2.7^{*}$ & $<0.005(\mathrm{HS})$ \\
\hline
\end{tabular}

NA: Not Applicable.

Table (10): Comparison between mass detection by digital mammography and tomosynthesis in dense breast (ACR C and D): Considering that mass as positive and non-mass as negative.

\begin{tabular}{llllll}
\hline $\begin{array}{l}\text { Mass } \\
\text { detection }\end{array}$ & $\begin{array}{c}\text { Digital } \\
\text { mammo- } \\
\text { graphy }\end{array}$ & $\begin{array}{c}\text { Tomosyn- } \\
\text { thesis }\end{array}$ & $\mathrm{X}^{2}$ & $p$ \\
\hline True positive & $22(35.5 \%)$ & $47.0(\%)$ & 22.3 & $<0.001$ (HS) \\
False positive & $0(0.0 \%)$ & $0(0.0 \%)$ & $\mathrm{NA}$ & $\mathrm{NA}$ \\
True negative & $10(16.1 \%)$ & $10(\%)$ & $\mathrm{NA}$ & $\mathrm{NA}$ \\
False negative & $30(48.4 \%)$ & $5(6.2 \%)$ & 27.6 & $<0.001(\mathrm{HS})$ \\
Sensitivity & $42.3 \%$ & $90.4 \%$ & $5.6^{*}$ & $<0.001$ (HS) \\
Specificity & $100 \%$ & $100 \%$ & $\mathrm{NA}$ & $\mathrm{NA}$ \\
Accuracy & $51.6 \%$ & $92 \%$ & $5.1^{*}$ & $<0.001$ (HS) \\
\hline
\end{tabular}

NA: Not Applicable.
Table (11): The distribution of different pathological entities "benign and malignant lesions" regarding final diagnosis either by histo-pathological evaluation or follow-up.

\begin{tabular}{lll}
\hline Pathological entities & $\mathrm{N}$ & $\%$ \\
\hline Benign: & 56 & \\
Fibrocystic changes. & 23 & 41.1 \\
Abscess & 1 & 1.8 \\
Granulomatous mastitis & 2 & 3.6 \\
Duct ectasia & 2 & 3.6 \\
Fibroadenoma & 17 & 30.3 \\
Benign phylloids & 1 & 1.8 \\
Normal & 7 & 12.5 \\
Postoperative scar & 3 & 5.4 \\
Malignant: & 40 & \\
Invasive ductal carcinoma & 28 & 70 \\
Invasive lobular carcinoma & 7 & 17.5 \\
Mucinous carcinoma & 2 & 5 \\
DCIS & 3 & 7.5 \\
\hline
\end{tabular}

Table (12): BIRADS scoring of indeterminate lesions detected by digital mammography comparing with BIRADS scoring of lesions detected by tomosynthesis.

\begin{tabular}{|c|c|c|c|c|c|c|}
\hline \multirow[t]{2}{*}{ BIRADS } & \multicolumn{2}{|c|}{$\begin{array}{c}\text { Digital } \\
\text { mammography }\end{array}$} & \multicolumn{2}{|c|}{$\begin{array}{l}\text { Tomosyn- } \\
\text { thesis }\end{array}$} & \multirow[t]{2}{*}{ MN } & \multirow[t]{2}{*}{$p$} \\
\hline & No. & $\%$ & No. & $\%$ & & \\
\hline 0 & 4 & 4.7 & 0 & 0.0 & Fisher & 0.1 \\
\hline I & 0 & 0.0 & 7 & 7.3 & Fisher & 0.1 \\
\hline II & 0 & 0.0 & 26 & 27.1 & Fisher & $<0.001(\mathrm{HS})$ \\
\hline III & 37 & 43 & 22 & 23 & 3.6 & $0.003(\mathrm{~S})$ \\
\hline IV a & 36 & 41.9 & 6 & 6.2 & 24.0 & $<0.001$ (HS) \\
\hline IV b & 3 & 3.5 & 5 & 5.2 & Fisher & 0.1 \\
\hline IV c & 6 & 7 & 13 & 13.5 & Fisher & $<0.001(\mathrm{HS})$ \\
\hline V & 0 & 0.0 & 17 & 17.7 & Fisher & $0.003(\mathrm{~S})$ \\
\hline
\end{tabular}

Table (13): Comparison between diagnostic performance of digital mammography and tomosynthesis in non dense breast (ACR A and B categories).

\begin{tabular}{|c|c|c|c|c|}
\hline $\begin{array}{l}\text { Final Digit } \\
\text { diagnosis }\end{array}$ & $\begin{array}{l}\text { tal Ton } \\
\text { gray }\end{array}$ & $\begin{array}{c}\text { hosyn- } \\
\text { thesis }\end{array}$ & $\chi^{2}$ & \\
\hline True positive & $10(29.4 \%)$ & $14(41.1 \%)$ & 0.6 & 0.4 \\
\hline False positive & $6(17.6 \%)$ & $1 \quad(3 \%)$ & Fisher & 0.1 \\
\hline True negative & $13(38.2 \%)$ & $18(52.9 \%)$ & 0.9 & 0.3 \\
\hline False negative & $5(14.7 \%)$ & $1 \quad(3 \%)$ & Fisher & 0.2 \\
\hline Sensitivity & $66.7 \%$ & $93.3 \%$ & $2.7^{*}$ & $<0.005$ (HS) \\
\hline Specificity & $68.4 \%$ & $94.7 \%$ & $2.8^{*}$ & 0.004 (HS) \\
\hline PPV & $62.5 \%$ & $93.3 \%$ & $3.06^{*}$ & $<0.002$ (HS) \\
\hline NPV & $72.2 \%$ & $94.7 \%$ & $2.4^{*}$ & $<0.01(\mathrm{~S})$ \\
\hline Accuracy & $67.6 \%$ & $94.1 \%$ & $2.8^{*}$ & $<0.004$ (HS) \\
\hline
\end{tabular}

Positive: Malignant.

Negative: Benign or no detected abnormality. 
Table (14): Comparison between diagnostic performance of digital mammography and tomosynthesis in dense breast (ACR C and D categories).

\begin{tabular}{lclll}
\hline $\begin{array}{l}\text { Final } \\
\text { diagnosis }\end{array}$ & $\begin{array}{c}\text { Digital } \\
\text { mammography }\end{array}$ & Tomosynthesis & $\chi^{2}$ & $p$ \\
\hline True positive & $15(24.2 \%)$ & $22(35.5 \%)$ & 1.3 & 0.2 \\
False positive & $17(27.4 \%)$ & $3(4.8 \%)$ & Fisher & 0.001 (S) \\
True negative & $20(32.3 \%)$ & $34(54.9 \%)$ & 5.5 & 0.02 (S) \\
False negative & $10(16.1 \%)$ & $3(4.8 \%)$ & Fisher & 0.07 \\
Sensitivity & $60.0 \%$ & $88.0 \%$ & $3.5^{*}$ & $<0.001$ (HS) \\
Specificity & $54.1 \%$ & $92.0 \%$ & $4.7^{*}$ & $<0.001$ (HS) \\
PPV & $47.0 \%$ & $88.0 \%$ & $4.8^{*}$ & $<0.001$ (HS) \\
NPV & $66.7 \%$ & $92.0 \%$ & $2.8^{*}$ & $<0.001$ (HS) \\
Accuracy & $56.5 \%$ & $90.3 \%$ & $3.8^{*}$ & $<0.001$ (HS) \\
\hline
\end{tabular}

Positive : Malignant.

Negative : Benign or no detected abnormality.

S : Significant.

HS : High Significant.

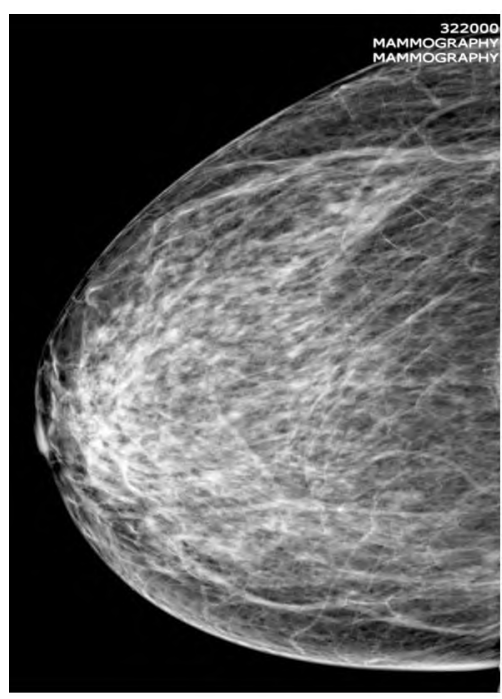

(A)

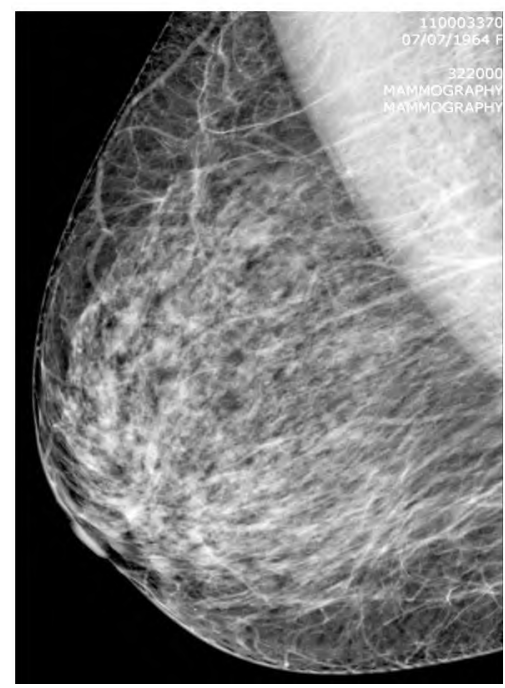

(C)

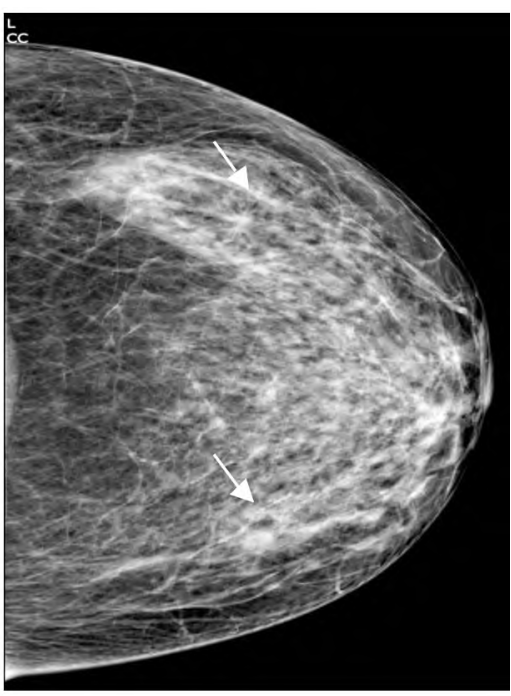

(B)

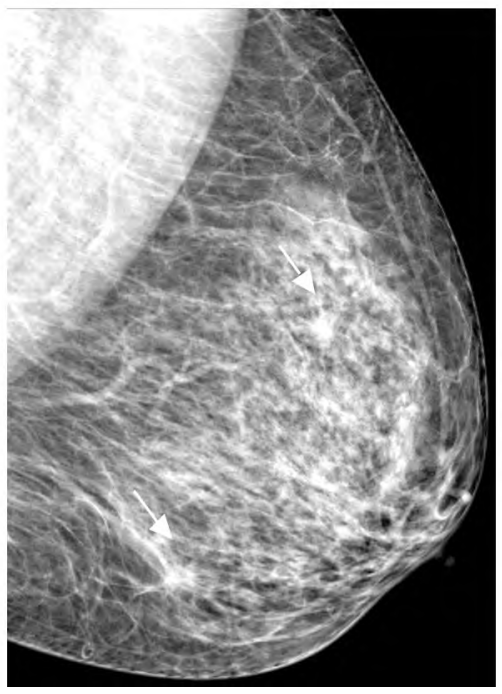

(D)

Fig. (1): Mammography CC and MLO views. 
Fig. (2): Tomosynthesis CC and MLO of the left breast.

Fig. (3): Mammography CC and MLO views.
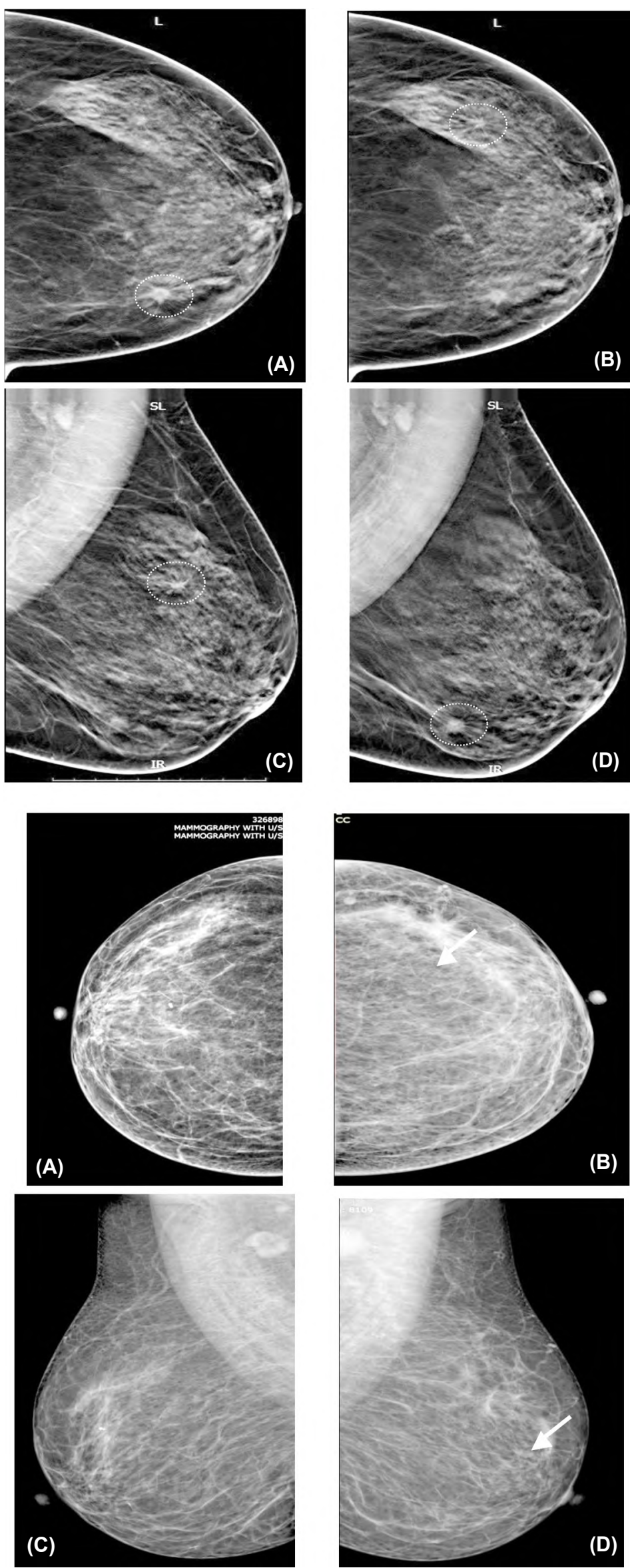


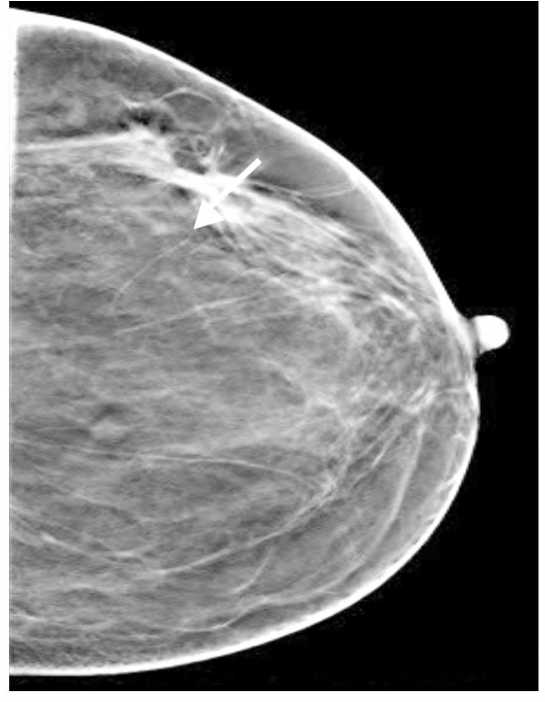

(A)

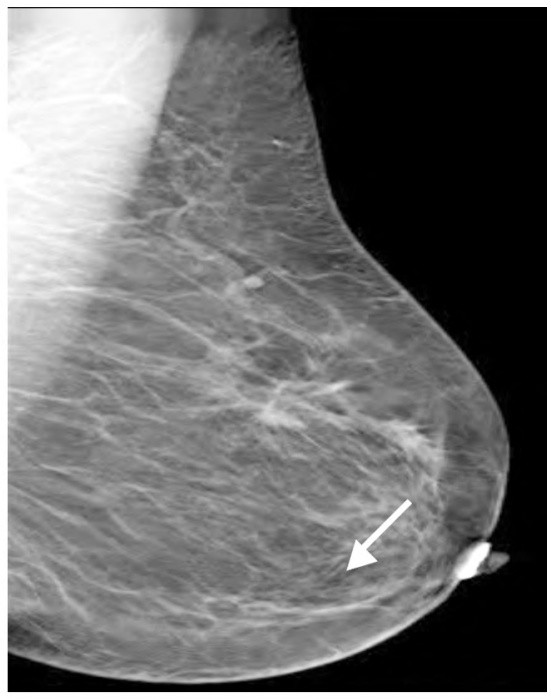

(B)

Fig. (4): Tomosynthesis CC and MLO of left breast.
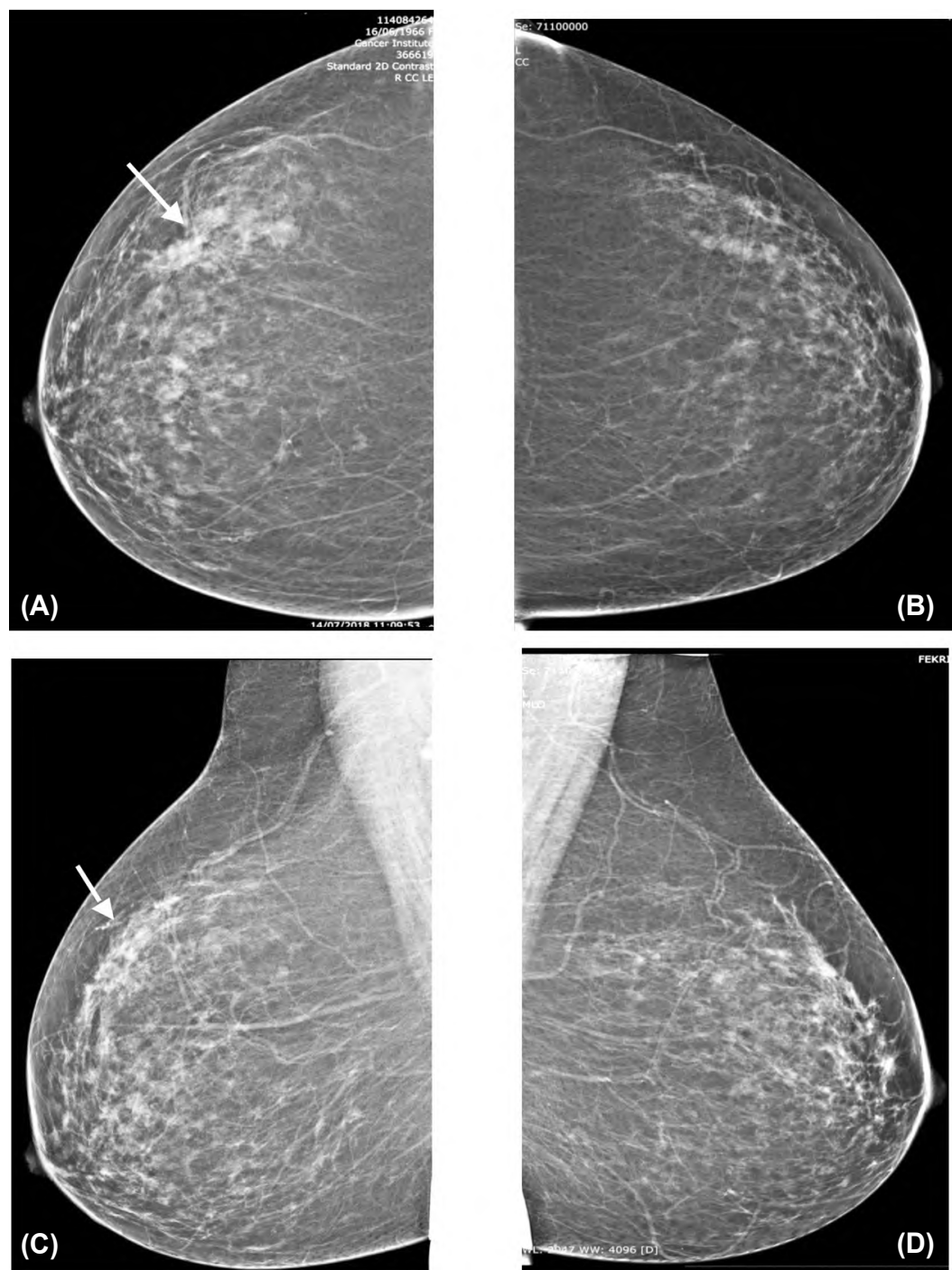

Fig. (5): Mammography CC and MLO views. 


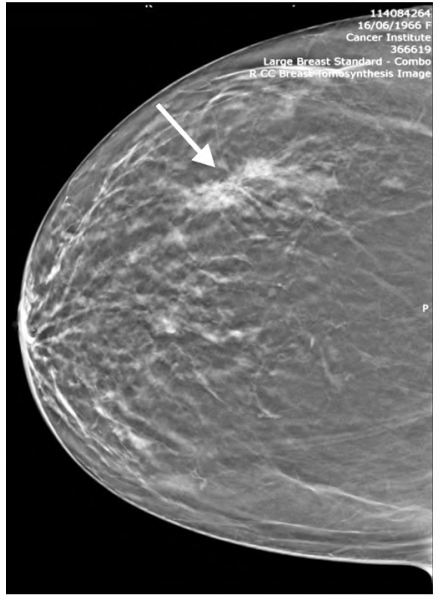

(A)

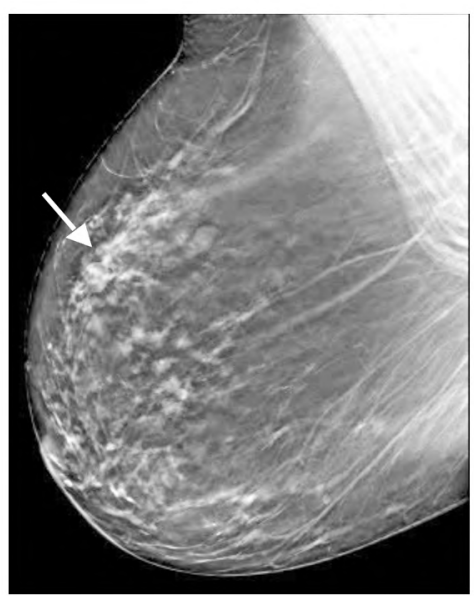

(B)

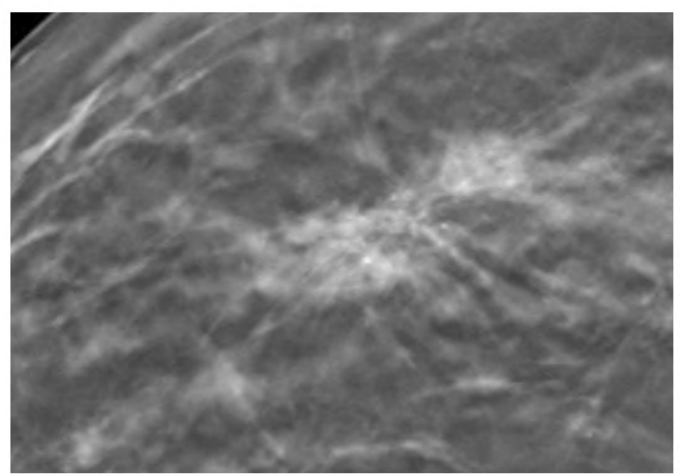

(C)

Fig. (6): Tomosynthesis CC and MLO views.

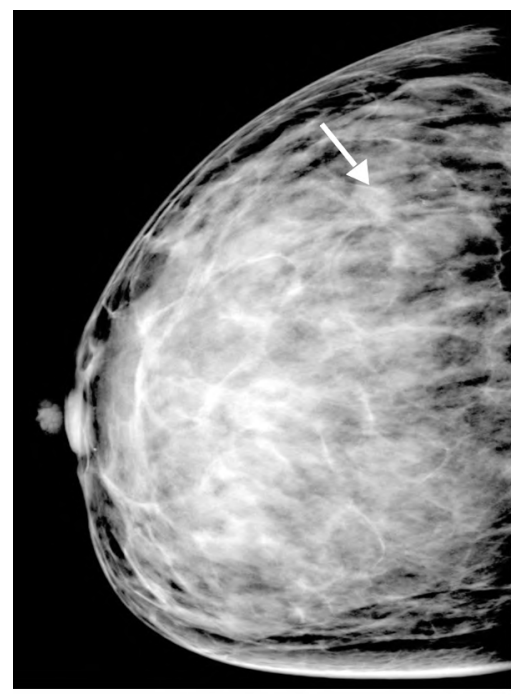

(A)

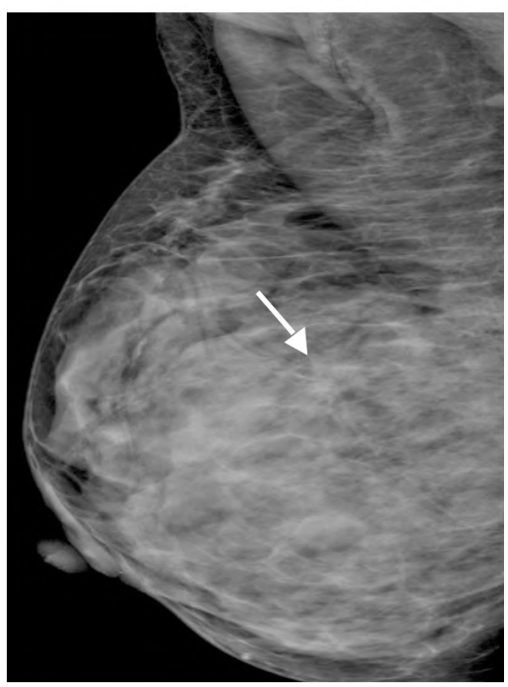

(C)

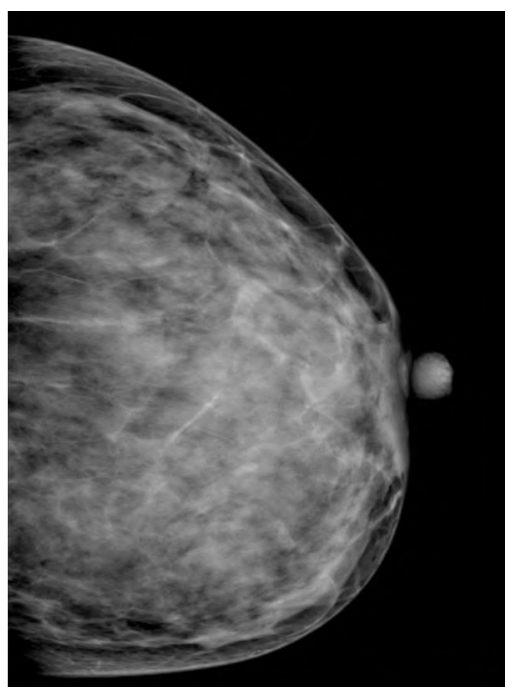

(B)

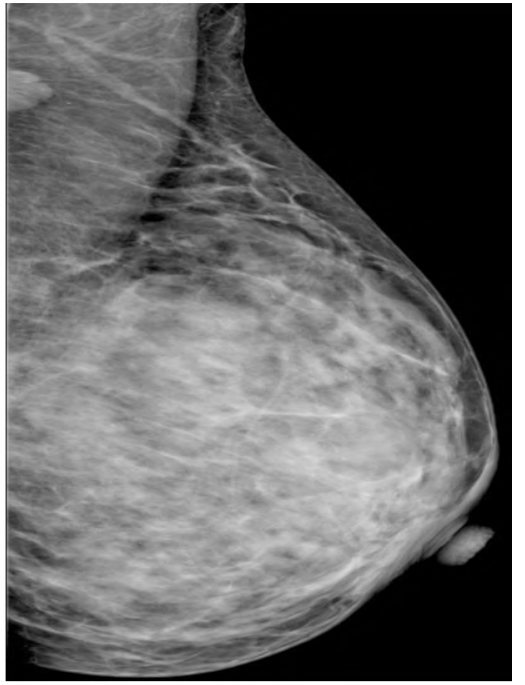

(D)

Fig. (7): Mammography CC \& MLO views. 

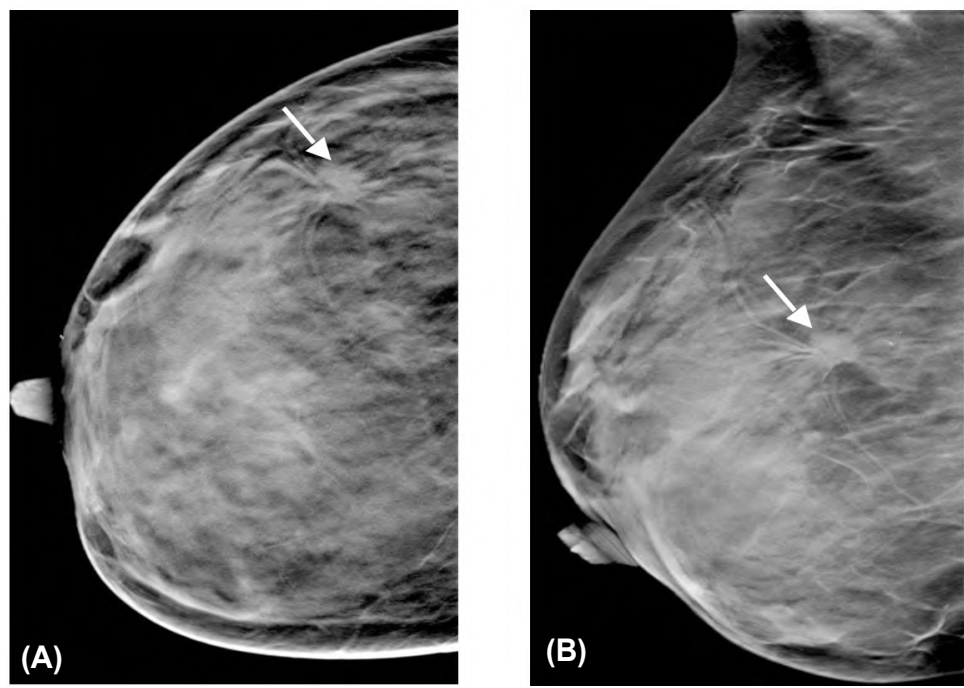

Fig. (8): Tomosynthesis of the right breast CC \& MLO views.
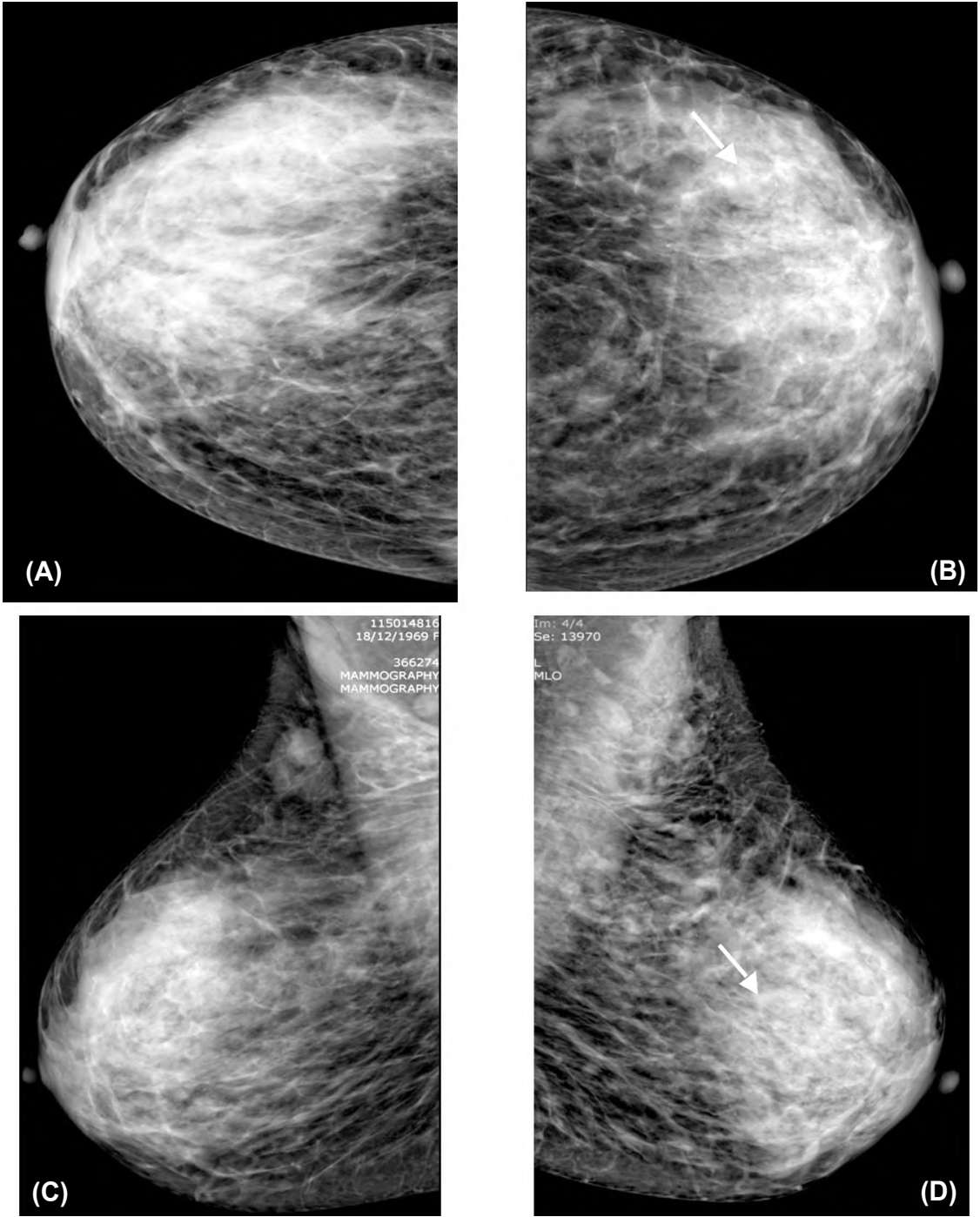

Fig. (9): Mammography CC \& MLO views. 


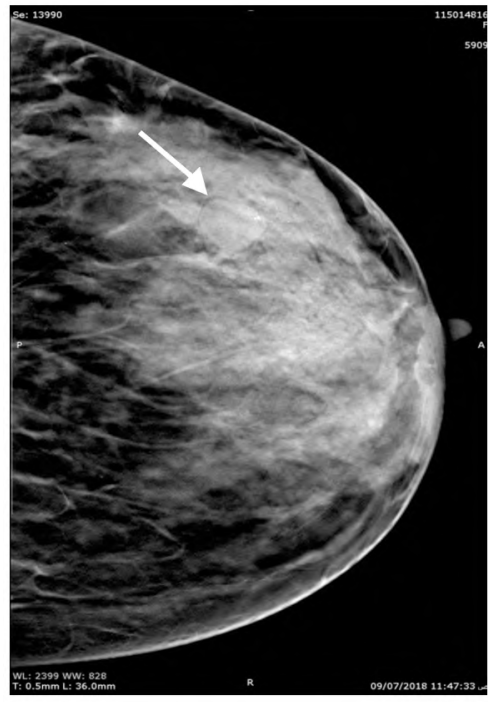

(A)

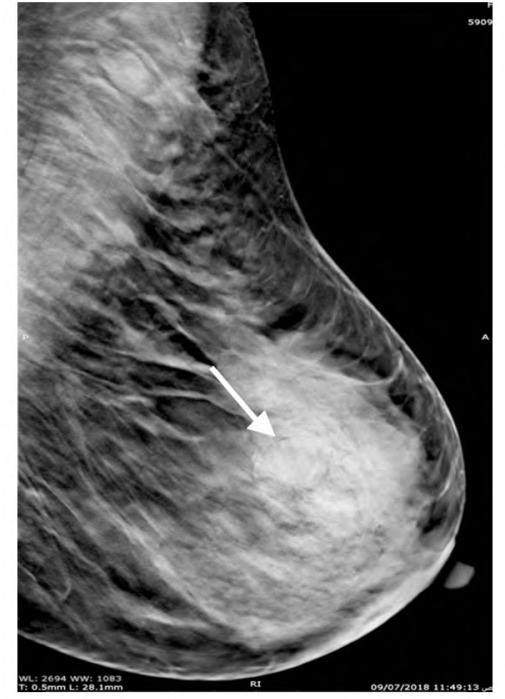

(B)

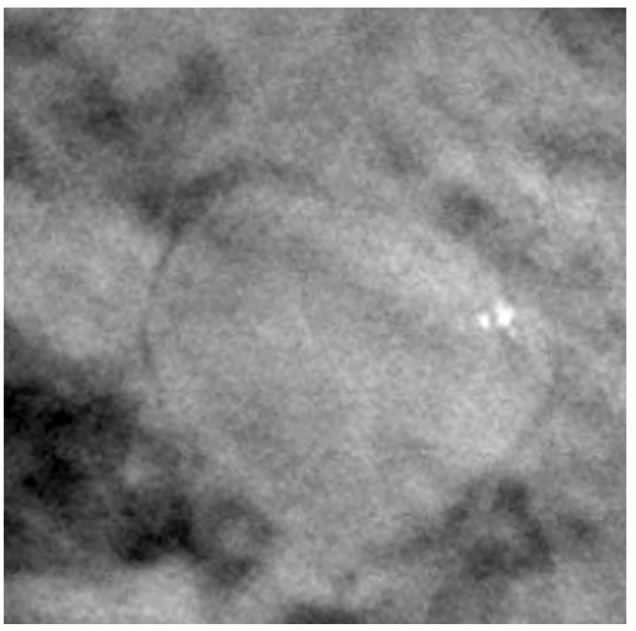

(C)

Fig. (10): Tomosynthesis of left breast CC \& MLO views.
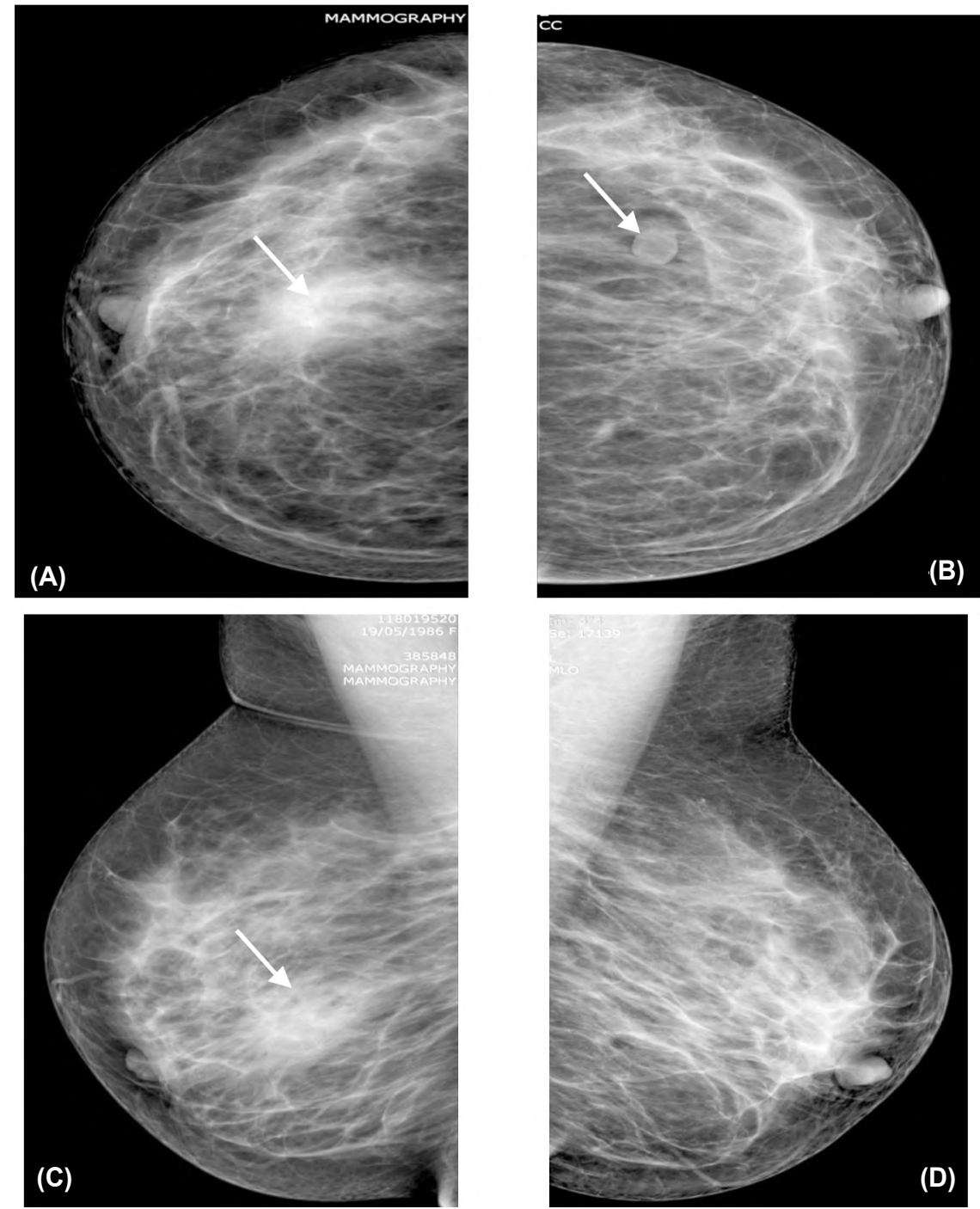

Fig. (11): Mammography MLO and CC view. 

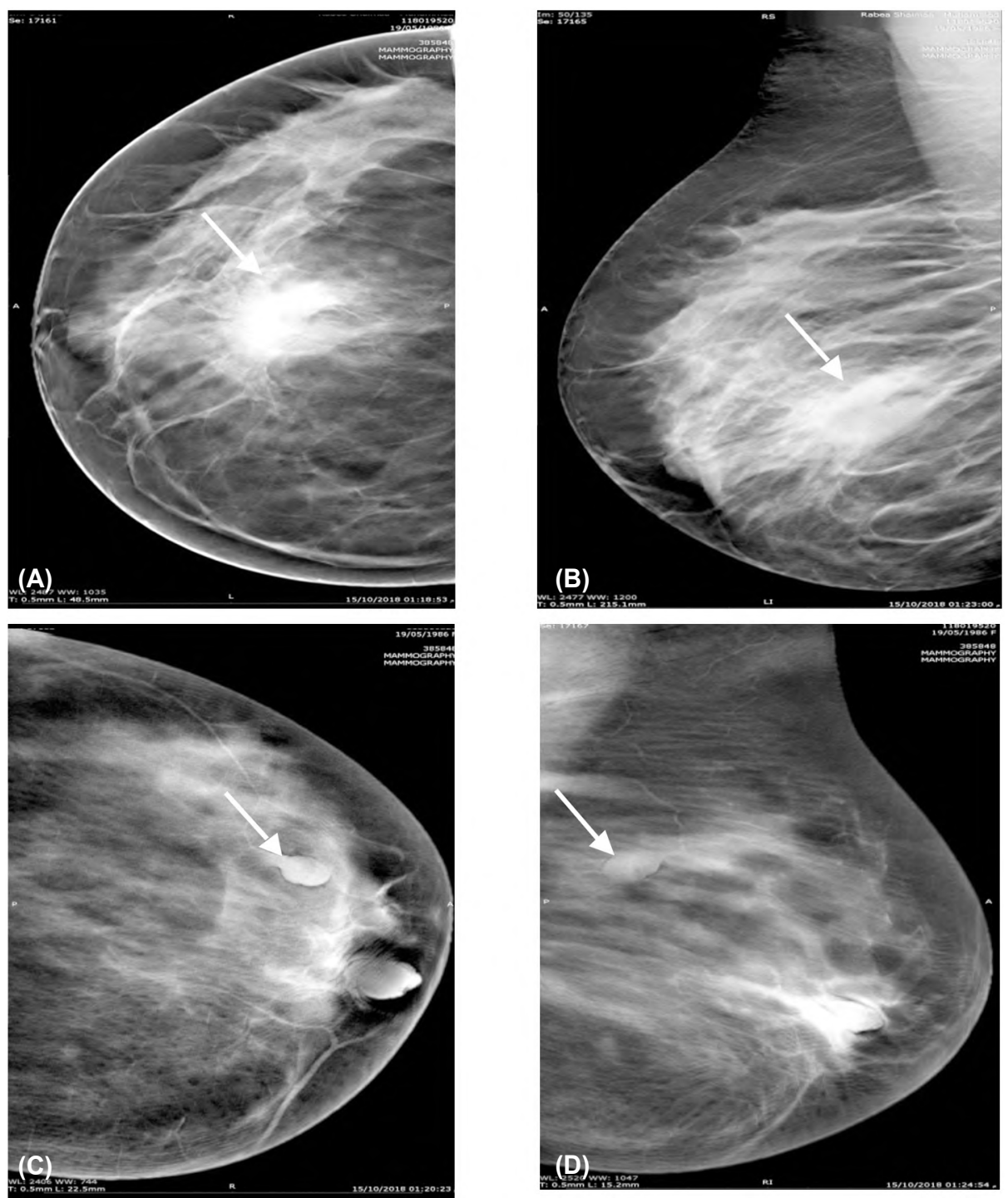

Fig. (12): Tomosynthesis MLO and CC views.

\section{Discussion}

Digital Breast Tomosynthesis (DBT) is a new diagnostic technique in breast evaluations with a promising role in rising sensitivity and specificity of Digital Mammography (DM) [6]

Women with extensive breast density are at higher risk of developing breast cancer; that cancer will not be detected because of masking of the radiological signs of cancer by increased density. Fatty breasts on mammogram allow better detection of breast cancer. Diagnosis of breast carcinoma is a complex issue and requires multiple imaging modalities [7].

Digital Breast Tomosynthesis (DBT) improves detection and characterization of breast lesions especially in women with dense breasts. It can overcome the inherent limitations of mammo- graphy due to overlapping of normal and pathological tissues [8] . 3D DBT allow better detection of lesion size, characterization of the lesions. It shows more screening sensitivity and decrease in recall rates [9],

In our study we evaluated the accuracy of DBT in detection and characterization of mammographically indeterminate breast lesions BIRADS 0 , III and IV.

In our study, Digital Breast Tomosynthesis (DBT) detected new 10 lesions not seen in digital mammography. It overcomes the problem of breast densities in DM and detected 36 masked masses not seen in DM. We found $3(7.5 \%)$ lesions with benign morphology on mammography proved to be irregular on Tomosynthesis, while $3(7.5 \%)$ lesions were described as irregular and proved to show benign shape morphology. Regarding the 
margin of mass lesions; Tomosynthesis changed the identified margin in $12(27 \%)$ mass lesions. So Tomosynthesis is better in margin and shape characterization as it overcome tissue overlap. The use of DBT allows proper BIRADS categorization and reduces unnecessary biopsies.

Helvie et al., (2010) reported that tomosynthesis improves characterization of malignant lesions compared to mammography. It allows more accurate assessment of shape and margin of the lesions [9]. They also reported that DBT revealed $77 \%$ of the perimeter of visible mass while conventional mammography showed $53 \%$ of the perimeter of the mass [9].

In a retrospective study done by Yang et al., 2013 [8]; stated that breast cancer cases were better described by DBT due to better lesion detection regarding shape and margin of masses and also subtle spiculated margins can be depicted by the use of thin slide.

Skaane, 2012 [10] also verified that using DBT enables better assessment of shape and margin of breast lesions due to elimination of overlapping tissue. It can distinguish superimposed tissue from breast lesions with increased specificity as compared with conventional mammography.

In our study, mammography detected 40 (41.7\%) masses while Tomosynthesis detected 76 $(79.2 \%)$ masses. Our results show significant difference between tomosynthesis and digital mammography in detection of masses $(p<0.001)$ as the sensitivity of DM for mass detection was $49 \%$ and accuracy $56.3 \%$ while in DBT sensitivity for mass detection was $92.7 \%$ and accuracy $93.7 \%$.

We found that in non-dense breast, DBT increases the sensitivity for mass detection from $70 \%$ to $96.7 \%$ and accuracy from $\% 73.5$ to $97.1 \%$ while in dense breast DBT raised sensitivity for mass detection from $42.3 \%$ to $90.4 \%$ and accuracy from $51.6 \%$ to $92 \%$, so the difference in sensitivity and accuracy for mass detection between DBT and DM more in dense breast than non-dense breast.

Waldherr et al., 2013, found that breast tomosynthesis revealed higher sensitivity and significantly (15-20\%) better NPV compared with FFDM regarding detection of masses, lesion margins and improved demarcation of radial distortions. Elevated NPV reduces recall rate especially in screening [12]

In this study, Tomosynthesis overcome the tissue overlap in focal asymmetries and can verify mass from overlapped fibro-glandular tissue. This was similar to study done by Christoph et al., 2013 [13] .

Skaane, 2012 [10] suggested that digital breast Tomosynthesis improves detection of breast architectural distortion particularly in cases with heterogeneous dense breasts. In our study, there were $56(58.3 \%)$ benign lesions and $40(41.7 \%)$ malignant lesions. Within the 56 benign lesions $41.1 \%$ of them were fibrocystic changes, $1.8 \%$ was breast abscess, $3.6 \%$ were diagnosed as granulomatous mastitis, $3.6 \%$ were duct ectasia, $30.3 \%$ were fibroadenoma, $1.8 \%$ were benign phylloids, $12.5 \%$ were diagnosed as normal and $5.2 \%$ were postoperative scaring. Giovanna Mariscotti et al., 2017 [14] reported 89 benign lesions; $25 / 89(28.1 \%)$ of lesions were fibroadenomata and 21/89 (23.5\%) as fibrocystic changes. Also close to Asbeutah A.M., et al., 2018 [15] who stated that fibroadenoma and fibrocystic changes were the most common benign lesions. The use of DBT may be helpful in reducing biopsies done for benign lesions [16]

In the present study malignant lesions was $(41.7 \%)$ of the total cases, $70 \%$ of them were invasive ductal carcinoma, $17.5 \%$ were invasive lobular carcinoma, 5\% mucinous carcinoma and $7.5 \%$ were DCIS and most common malignant tumor was invasive ductal carcinoma (70\%). This was similar to Ali et al., 2015 [17] who reported that out of the total 67 malignant lesions out of 145 breast lesions. Invasive ductal cancers were $34(50.8 \%)$ lesions. Also Förnvik et al., 2010 [18] stated that invasive ductal cancers is the most common malignant tumor.

In our study, we found that tomosynthesis clarified 10 more lesions hardly seen in digital mammography and changed the identified BIRADS category in $64(74.4 \%)$ lesions. It upgraded 30 $(34.9 \%)$ lesions and downgraded $34(39.5 \%)$ lesions. Tomosynthesis significantly decreased the number of indeterminate lesions (BIRADS III, IVa) from $84.9 \%$ to only $29.2 \%$.

Hakim et al., 2010 [19] found that combined FFDM and DBT were more accurate than additional mammographic views particularly in cases with known masses, architectural distortions, or asymmetries. In this study; 3D Digital Breast Tomosynthesis showed better lesion BIRADS classification and significantly decreased the number of indeterminate/suspicious lesions, (BIRADS $3 \& 4$ ).

Yang et al., 2013 [8] reported that tomosynthesis upgraded $85.2 \%$ of lesions from BIRADS 2 or 3 to BIRADS 4 or 5 in non-calcified lesions, upgrad- 
ed $78.2 \%$ of all breast lesions to equal or higher than BIRADS $4 \mathrm{~b}$, with only mild to moderate improvement of the diagnostic accuracy.

Also, in concordant with Raghu et al., 2016 [20] who stated that tomosynthesis significantly decrease number of indeterminate lesion BIRADS III from $33.3 \%$ in DM to only $16.4 \%$ by DBT with $p<0.0001$. But they reported no change in BIRADS category (4 and 5) after addition tomosynthesis.

In our study we compared changing in BIRADS category between tomosynthesis and digital mammography in each breast density we found that in ACR B; 12 cases (41.4\%) showed the same diagnosis by DM and DBT while 17 cases $(58.6 \%)$ either upgrades or downgrades and in ACR C; 7 cases $(15.2 \%)$ showed the same diagnosis by both DM and DBT while 39 cases either upgrades or downgrades $(84.8 \%)$. So, change in BIRADS grading after addition of DBT more in ACR C than B.

Rangarajan, et al., 2016 [21] also reported that in ACR A and B (80.9\% and $81.5 \%$ respectively) of cases there were no change in BIRADS categorization after addition of DBT and in ACR C and $\mathrm{D}$ (64\% and $57.5 \%$, respectively), while in dense breast (ACR C and D) (77.3\%) of cases there were superior categorization.

Waldherr, et al., 2013 [12], in a study comparing the role of Mammography and Tomosynthesis in the diagnostic work up showed that digital mammography revealed sensitivity $70.5 \%$, specificity $80.8 \%$, PPV $86.1 \%$ and NPV $61.8 \%$.

Tamaki, et al., 2012 [22] performed a retrospective analysis of mammography findings in 1267 Japanese women. They calculated sensitivity, specificity and positive predictive value were 92.8 , 31.4 and $63.1 \%$, respectively.

Our study; on comparing imaging findings with the pathology results, $36(37.5 \%)$ lesions were true positives, $5(5.2 \%)$ lesions were false positive, 4 (4.2\%) lesions were false negatives and 51 (53.1\%) lesions were true negatives. The false positive results are less (5 instead of 23 case) when compared to digital mammography. Tomosynthesis overcame the tissue overlap in focal asymmetries and was able to verify if there is an underlying mass and whether single or multiple masses.

In our study we found that Tomosynthesis had a sensitivity of $90 \%$, a specificity of $91.1 \%$, a positive predictive value of $88 \%$, a negative predictive value of $92.7 \%$ and accuracy $91 \%$. Waldherr, et al., 2013 [12], in a study comparing the role of
Mammography and Tomosynthesis; showed that Digital Breast Tomosynthesis had sensitivity $84 \%$, specificity $83.9 \%$, positive predictive value $89.4 \%$ and negative predictive value $76.5 \%$.

Elizalde et al., 2014 [23] reported that sensitivity of DM after addition of tomosynthesis increase from $69.05 \%$ by DM alone to 86.9 by combination of both modalities. But they found that specificity decrease after addition of DBT from $88.2 \%$ to $83.5 \%$, as BIRADS 3 lesions were considered as positive and this is a possible explanation for the lower specificity of additional DBT in this study.

Lei et al., 2014 [24] reported that DBT has a higher sensitivity and specificity in breast diagnosis than DM. The sensitivity and specificity of DBT as $90.0 \%$ and $79.0 \%$, and for DM they were $89.0 \%$ and $72.0 \%$, respectively.

In our study we found that sensitivity of mammography decrease with increase breast density due to masking of glandular tissue to underlying lesion, the calculated sensitivity was $60 \%$ in dense breast (ACR C and D) compared to $66.7 \%$ in nondense breast (ACR A and B) while adding tomosynthesis increase sensitivity in each breast density with decrease false positive results.

Similar to Niell et al., 2017 [25] which stated that the sensitivity of mammography decreases in women with dense breast, measuring $30 \%$ to $64 \%$ for extremely dense breasts compared with $76 \%$ to $98 \%$ for fatty breasts. Addition of Tomosynthesis to FFDM increased the invasive cancer detection rate by $40 \%$ and decreased false positives by $15 \%$, compared with FFDM alone.

In our study we found that adding DBT to digital mammography in non-dense breast (ACR $A$ and $B$ ) raised sensitivity for detection of malignant lesions from $66.7 \%$ to $93.3 \%$, specificity from $68.4 \%$ to $94.7 \%$, PPV from $62.5 \%$ to $93.3 \%$, NPV from $72.2 \%$ to $94.7 \%$ and accuracy from $67.6 \%$ to $94.1 \%$, while in dense breast (ACR C and D) raised sensitivity from $60 \%$ to $88 \%$, specificity from $54.1 \%$ to $82.2 \%$, PPV from $47 \%$ to $88 \%$, NPV from $66.7 \%$ to $92 \%$ and accuracy from $56.5 \%$ to $90.3 \%$, so the difference in sensitivity, specificity, PPV, NPV and accuracy between DBT and DM in dense breast (ACR C and D) was more than in non-dense breast (ACR A and B).

In a study done by Waldherr et al., 2013 they stated that digital breast tomosynthesis was more sensitive than FFDM in both dense and fatty breasts. This can be explained in some patients by 
the ability of digital breast tomosynthesis to better delineate radial distortions of low-density lesions, better demarcate small lesions, and better assessment of lesion margins. The lesion margin is one of the key factors in determining whether a lesion is scored as benign or malignant [12].

In this study, we found that Tomosynthesis separates overlapping tissue in dense breast by acquisition of multiple images over limited angular range. Tomosynthesis showed higher sensitivity and specificity and diagnostic accuracy than Mammography as it allows better detection of breast cancer, characterization of lesions, better margin assessment of masses and decrease false positive recall rate.

\section{Conclusion:}

We concluded that DBT showed higher sensitivity and specificity and diagnostic accuracy than Mammography as it allows better detection and characterization of breast lesions with decrease of false positive and negative cases.

\section{References}

1- ZEENELDIN A.A., RAMADAN M., GABER A.A., et al.: Clinicopathological features of breast carcinoma in elderly Egyptian patients: A comparison with the nonelderly using population-based data. J. Egypt Natl. Canc. Inst., 25 (1): 5-11, 2013.

2- CHANG R.F., CHEN C.J., CHIOU K.Y., MOON W.K., et al.: Breast Lesion Classification Using 3-D Ultrasound. In: Suri J.S., Kathuria C., Chang R.F., et al., editors Advances in Diagnostic and Therapeutic Ultrasound Imaging. Artech House USA, 142-64, 2008.

3- VAN DEN BIGGELAAR F.J., KESSELS A.G., VAN ENGELSHOVEN J.M., et al.: Strategies for digital mammography interpretation in a clinical patient population. Int. J. Cancer, 125 (12): 2923-9, 2009.

4- DROMAIN C. and BALLEYGUIER C.: Digital Mammography in Ulrich Bick, Felix Diekmann editors, London Springer Science and Business Media introduction 5 th edition, 12.1 (188), 2010.

5- FALLENBERG E.M., DROMAIN C., et al.: Contrast enhanced spectral mammography versus MRI: Initial results in the detection of breast cancer and assessment of tumour size. Eur. Radiol., 24 (1): 256-64, 2014.

6- BUNOVIC N.P., PRVULOVIC M., DJILAS D., et al.: The value of breast tomosynthesis in the assessment of BIRADS 3 lesions. European society of radiology, ECR/C1905,2014

7- SLEEBA T., SUBAPRADHA A., RAMACHANDRAN M., et al.: Role of dual-energy contrast-enhanced digital mammography as a problem-solving tool in dense breasts: A case report. Indian J. Radiol. Imaging, 23 (3): 219-22, 2013.
8- YANG T., LIANG H., CHOUC C., HUANG J., et al.: Adjunctive Digital Breast Tomosynthesis in Diagnosis of Breast Cancer. Biomed. Res. Int., 597253, 2013.

9- HELVIE M.: Digital Mammography Imaging: Breast Tomo-synthesis and Advanced Applications. Radiol. Clin. North Am., 48 (5): 917-29, 2010.

10- SKAANE P.: Tomosynthesis in X-ray: Proven additional value? Elsevier, Eur. J. Radiol., 1: S 156-7, 2012.

11- HAWLEY J.R, M.D., JUSTINE K. KANG-CHAPMAN, M.D., SARAH E. BONNET, M.D., et al.: Diagnostic Accuracy of Digital Breast Tomosynthesis in the Evaluation of Palpable Breast Abnormalities, Acad. Radiol.: Article in Press, 2017.

12- WALDHERR C., CERNY P., ALTERMATT H.J., et al.: Value of one view breast tommosynthesis versus two view mammography in diagnostic workup of women with clinical signs and symptoms and in women recalled from screening; AJR, 200 (1): 226-31, 2013.

13- CHRISTOPH I. LEE and CONSTANCE D. LEHMAN: Digital Breast Tomosynthesis and the Challenge of Implementing an Emerging Breast Cancer Screening Technology Into Clinical Practice. J. Am. Coll. Radiol., 10: 913-917. Copyright () American College of Radiology, 2013.

14- GIOVANNA MARISCOTTI, MANUELA DURANDO, NEHMAT HOUSSAMI, et al.: Comparison of synthetic mammography, reconstructed from digital breast tomosynthesis, and digital mammography: Evaluation of lesion conspicuity and BI-RADS assessment categories, Breast Cancer Res. Treat., 166: 765-73, 2017.

15- ASBEUTAH AKRAM M., NOURALHUDA KARMANI, ABDUL AZIZ A. ASBEUTAH, et al.: Comparison of Digital Breast Tomosynthesis (DBT) and Digital Mammography (DM) for Detection of Breast Cancer in Women in Kuwait Medical Principles and Practice, 156.211: 148140,2018

16- POPLACK S., TOSTESON T., KOGEL C. and NAGY H.: Digital Breast Tomosynthesis: Initial Experience in 98 Women with Abnormal Digital Screening Mammography. American Journal of Roentgenology, 189: 616623, 2007

17- ALI T.F.T., ASMAA M.A. MAGID, MOHAMED A. TAWAB, et al.: Potential impact of tomosynthesis on the detection and diagnosis of breast lesions, The Egyptian Journal of Radiology and Nuclear Medicine; Article in Press, 2015

18- FÖRNVIK D., SOPHIA ZACKRISSON, OTTO LJUNGBERG, et al.: Breast tomosynthesis: Accuracy of tumor measurement compared with digital mammography and ultrasonography, Acta. Radiologica, 3: 141-5, 2010.

19- HAKIM C.M., DENISE M. CHOUGH, MARIE A. GANOTT, et al.: Digital Breast Tomosynthesis in the Diagnostic Environment: A Subjective Side-by-Side Review AJR, 195: 172-6, 2010.

20- RAGHU M., MELISSA A. DURAND, LIVA ANDREJEVA, et al.: Tomosynthesis in the Diagnostic Setting: Changing Rates of BI-RADS Final Assessment over Time, RSNA, 281 (1): 55-61, 2016. 
21- RANGARAJAN K., HARI S., THULKAR S., et al.: Characterization of lesions in dense breasts: Does tomosynthesis help? Indian J. Radiol. Imaging, 26: 210-5, 2016.

22- TAMAKI K., ISHIDA T., MIYASHITA M., et al.: Retrospective analysis of mammographic findings for Japanese women: A potential predictor for breast malignancies. Cancer Science, 103 (3): 472-6, 2012.

23- ELIZALDE A., LUIS PINA, JON ETXANO, et al.: Additional US or DBT after digital mammography: Which one is the best combination?, Acta. Radiol., 0 (0) 1-6, 2014.

24- LEI J., PIN YANG and LI ZHANG, et al.: Diagnostic accuracy of digital breast tomosynthesis versus digital mammography for benign and malignant lesions in breasts: A meta-analysis, Eur. Radiol., 24: 595-602, 2014.

25- NIELL B.L., PHOEBE E. FREER, ROBERT JARED WEINFURTNER, et al.: Screening for Breast Cancer, Radiol. Clin. N. Am., 55: 1145-62, 2017.

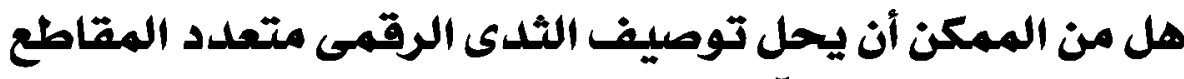

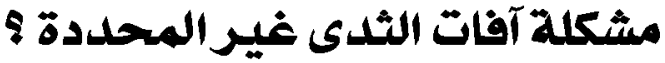

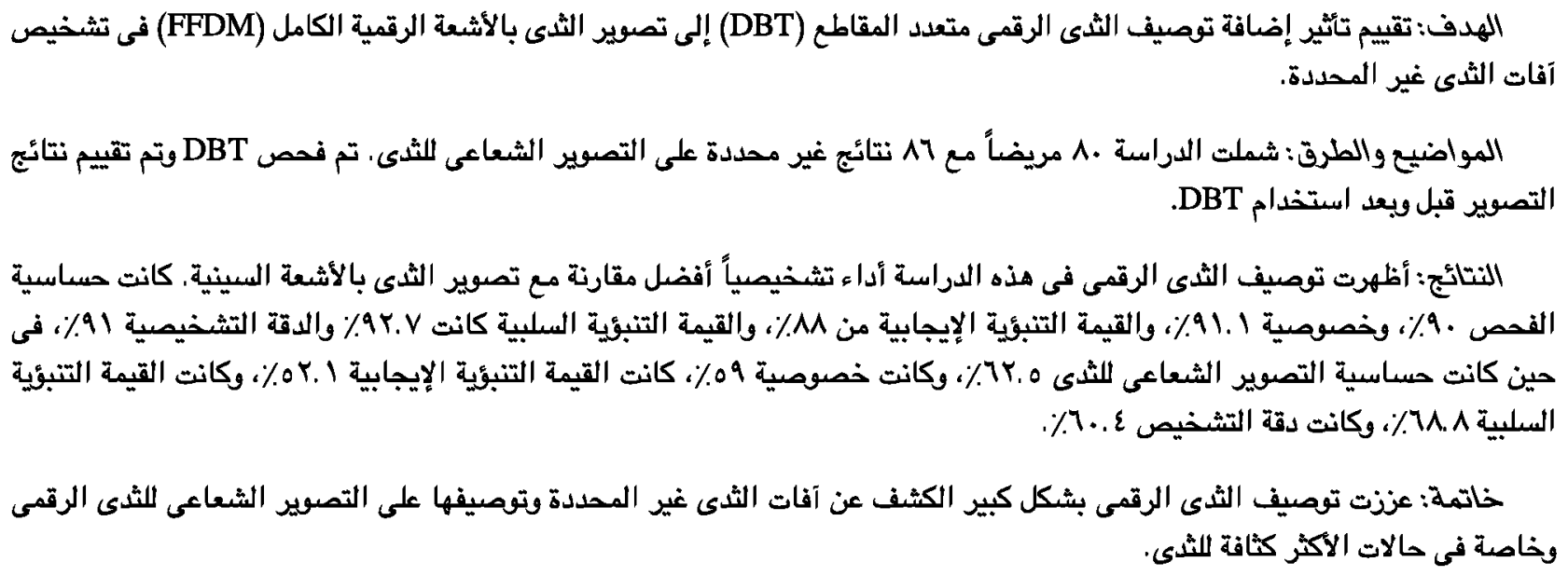

